\title{
Studies on Marker Assisted Background Screening of Sorghum Downy Mildew Resistant Introgressed Lines $\left(\mathrm{BC}_{3} \mathrm{~F}_{3}\right)$ in Maize
}

\author{
K. Sumathi ${ }^{1 *}$ K. N. Ganesan ${ }^{2}$ and N. Senthil ${ }^{3}$ \\ ${ }^{1}$ Centre for Plant breeding and Genetics, TNAU Coimbatore, India \\ ${ }^{2}$ Millet Breeding Station, TNAU Coimbatore, India \\ ${ }^{3}$ Centre for Plant Molecular Biology, TNAU Coimbatore, India \\ *Corresponding author
}

\section{A B S T R A C T}

\section{Keywords}

Back ground screening,SSR

Markers, Sorghum

Downy Resistant lines, Maize

Article Info

Accepted:

25 May 2018

Available Online:

10 June 2018
The present investigation was carried out at Centre for Plant Molecular Biology, Tamil Nadu Agricultural University, Coimbatore, Tamil Nadu, India. The main objective of this research is to retain the recipient parent genome outside the target gene with help of SSR markers. For this purpose, background screening was done in the selected four introgressed lines $\left(\mathrm{BC}_{3} \mathrm{~F}_{3}\right)$ viz.,UMI 79/936-C1-7-7-7-46-2 ,UMI 79/936-C1-7-7-7-80-17 ,UMI 79/936C1-7-7-7-92-1 and UMI 79/936-C1-7-7-7-92-7 with major QTLs for resistance to sorghum downy mildew in order to estimate the recovery of the genome of the recurrent parent. A total of 51 SSR markers, which showed distinct alleles between the parents, were selected for the genome wide background analysis. The graphical genotyping of the $\mathrm{BC}_{3} \mathrm{~F}_{3}$ SDMresistant introgressedlines revealed that the complete recovery of UMI 79genome was present in chromosome 3 in UMI 79/936-C1-7-7-7-46-2 and UMI 79/936C1-7-7-7-80-17.The complete recovery of UMI 79genome was present in chromosome 7 in UMI 79/936-C1-7-7-7-92-1 and UMI 79/936-C1-7-7-7-92-7.The genotype UMI 79/936C1-7-7-7-46-2 has revealed that the 92.45 per cent (maximum recovery) of the genome were derived from the recurrent parent UMI 79,genotype UMI 79/936-C1-7-7-7-80-17 andUMI 79/936-C1-7-7-7-92-7 showed that 89.68 per cent and 80.6 per cent ofrecovery respectively.

\section{Introduction}

Maize (Zea mays L.) is an important human food, livestock feed, and an industrial raw material.It is an important cereal in many developed and developing countries of the world. In terms of breeding, it is one of the most studied species and has been used as a model in manysituations. It is widely used for animal feed and industrial raw material in the developed countries where as the developing countries use it in general for feed. In Indian Agriculture, Maize occupies a prominent position and each part of the maize plant is put to one or the other use and nothing goes as waste. In India, about 28per cent of maize produced is used for food purpose, about 11 per cent as livestock feed, 48per cent as poultry feed, 12per cent in wet milling industry and 1per cent as seed (AICRP on 
Maize, 2007). However, demand for maize crop in the developing countries are expected to surpass the demand for both wheat and rice by the year 2020 (Prasanna and Hoisington, 2003).

In India, maize is grown in a wide range of environments, extending from extreme semiarid to sub-humid and humid regions. The crop is also very popular in the low- and midhill areas of the western and northeastern regions.In the tropics, grain is primarily grown for human consumption.The demand for maize as an animal feed will continue to grow faster than the demand for its use as a human food, particularly in Asia, where a doubling of production is expected from the present level of $165 \mathrm{Mt}$ to almost $400 \mathrm{Mt}$ in 2030. (Paliwal et al., 2000). Various biotic and abiotic stresses can constrain maize production with considerable yield loss.

Marker-assisted background selection a term coined by Hospital and Charcosset (1997) was initially proposed by Young and Tanksley (1989).This strategy has been used extensively in commercial maize breeding programmes, particularly for selection of lines carrying transgenes conferring herbicide tolerance or insect resistance ( $\mathrm{Yu}$ et al1996). Marker assisted background selection is the selection of individuals in advanced generations possessing the maximum genome of the recurrent parent. The main objective is to retain the recipient parent genome outside the target gene and the markers will help to identify the recurrent parent genome constitution of each back cross individual to select the best progeny for further advancement.

\section{Materials and Methods}

The experiments were conducted in Centre for Plant Molecular Biology, Tamil Nadu Agricultural University, Coimbatore, Tamil Nadu, India during December 2014. Four
$\mathrm{BC}_{3} \mathrm{~F}_{3} \mathrm{SDM}$ introgressed linesviz., UMI 79/936-C1-7-7-7-46-2 ,UMI 79/936-C1-7-7-780-17,UMI 79/936-C1-7-7-7-92-1 and UMI $79 / 936-C 1-7-7-7-92-7$ were used in the present study. It is derived from crossing the inbred UMI 79 which is susceptible for sorghum downy mildew and UMI 936(w) which has resistance for sorghum downy mildew and backcrossing progenies with UMI79.Selfing was done for two generation to get $\mathrm{BC}_{3} \mathrm{~F}_{3}$ Population. Background screening was done in the selected introgressed lines for SDM in order to estimate therecovery of the genome of recurrent parent.For this background survey, the polymorphic SSRmarker survey was carried out using 51 polymorphic SSR marker sequences obtained from maizegdbdatabase (www.maizegdb.org). These markers were located among the ten chromosomes ofmaize genome.The physical position of the polymorphic markers was obtained from the maizegdb database for each chromosome separately.Parental polymorphism for these two parents UMI79 and UMI 936(w)was already done.The markers details are given in Table.1.

\section{Results and Discussion}

Marker Assisted Backcrossing (MAB) combines 'Foreground' selection of donor alleles linked to markers and 'Background' selection of recurrent parent alleles in the later generation and become most efficient and feasible breeding approach for introgression of SDM resistant QTLs. Background markers are markers that are unlinked to the target gene/QTL on all other chromosomes, in other words, markers that can be used to select against the donor genome. This is extremely useful because the recurrent parent recovery can be greatly accelerated. With conventional backcrossing, it takesa minimum of six back cross generations to recover the recurrent parent andthere may still be several donor chromosome fragments unlinked to the target gene. 
Table.1 Skewness and Kurtosis observed in the SDM resistant progenies of BC2F1 generation

\begin{tabular}{|c|c|c|c|}
\hline Marker & Chrom. & Forward Primer & Reverse primer \\
\hline bnlg1178 & 1 & ACTACAGTTGAACGCCCCTG & GCTCATGTGCAAATGCAAGT \\
\hline umc2151 & 1 & ATATGTGGTATTTTCTGCAGGCGT & AAAATCCTATACAGAAAACGGGCG \\
\hline umc2234 & 1 & CAAGATCGTTAGGTTCTAGGCGTC & GACGGACTATAGAGGGCGATGAG \\
\hline umc2077 & 2 & AAACTCACTGAACATGATCCTGGC & CTGGTTCGGATGCAAGTAGTCAG \\
\hline phi127 & 2 & ATATGCATTGCCTGGAACTGGAAGGA & AATTCAAACACGCCTCCCGAGTGT \\
\hline umc2214 & 2 & ACCCCCTGATTCTCTCTTACGTTT & CTGGATGAGGAGGAAGAATACGAG \\
\hline umc1552 & 2 & CTCGATAGCTCTGCTGCTTCCTC & CAACACCAGCCCTACCCAGA \\
\hline umc1256 & 2 & CATCTCGACCTTTGACATTCTCCT & AGAAGACGATGATGATGATGCAGA \\
\hline bnlg197 & 3 & GCGAGAAGAAAGCGAGCAGA & CGCCAAGAAGAAACACATCACA \\
\hline umc1158 & 3 & AATGCAACTGCTTCAGCTCCTACT & CGACGAATCGAGAAAAGATATTTGA \\
\hline umc2101 & 3 & CCCGGCTAGAGCTATAAAGCAAGT & CTAGCTAGTTTGGTGCGTGGTGAT \\
\hline phi243966 & 3 & CGACCGAAACGAATCAAAA & TACTAGGCTGACACGCACG \\
\hline phi073 & 3 & GTGCGAGAGGCTTGACCAA & AAGGGTTGAGGGCGAGGAA \\
\hline umc1594 & 3 & CACTGCAGGCCACACATACATA & GCCAGGGGAGAAATAAAATAAAGC \\
\hline bnlg1035 & 3 & TGCTTGCACTGTCAGGAATC & CAGCTCTGACACACCACACA \\
\hline umc2263 & 3 & CGTGCTTATATGGGTTCTTGGGT & GTTTGGTTGCTGCGACCTCTT \\
\hline umc1608 & 3 & GTGTCGTGTTGGGAGAACATGAG & TAACTACTACACCACTCGCGCAAA \\
\hline umc1231 & 3 & CTGTAGGGCTGAGAAAAGAGAGGG & CGACAACTTAGGAGAACCATGGAG \\
\hline umc1136 & 3 & CTCTCGTCTCATCACCTTTCCCT & CTGCATACAGACATCCAACCAAAG \\
\hline umc1030 & 3 & TCCAGAGAATGAGATGACAAGACG & CAGAATAACAGGAGATGAGACGCA \\
\hline
\end{tabular}




\begin{tabular}{|c|c|c|c|}
\hline Marker & Chrom & Forward Primer & Reverse primer \\
\hline umc2104 & 3 & CTGCTGGCAGTGGCAGTATTC & TACTGCTACACCTTTGTCGTCACC \\
\hline phi053 & 3 & AACCCAACGTACTCCGGCAG & CTGCCTCTCAGATTCAGAGATTGAC \\
\hline bnlg420 & 3 & CTTGCGCTCTCCTCCCCTT & GGCCAGCTCACTGCTCACT \\
\hline umc 2360 & 4 & TAGCAGCTAGCTTCAGTCACAGGC & CAGATCGGACTACTGGTGGCTAAG \\
\hline umc1757 & 4 & ATAGGAGGTGAGGTGAGGAGGAAG & TTTTCTGCAGGGATAACATTTGTG \\
\hline BNLG1601 & 4 & ATCGTGCGCTAGTCCAGAGT & CAGACCAGAGACCATCTGCA \\
\hline umc 2365 & 4 & GAAATCCATTCATTCCTTCGTCC & GTGACCTCTAGCTAGCTGGGCTATT \\
\hline umc2136 & 5 & CCAGATGCGGAAGTAGACGG & GATTCGGAGGTGATCTGACCTGT \\
\hline umc1056 & 5 & CGGATCGCTTTTTACCGTCTATAA & AGCAAGAGTAGCGTTCCATTTCAG \\
\hline umc2298 & 5 & ATCCACTCCCAAGTCCCAACAC & CTTCTTCCGGTTCTTCTTCAGGC \\
\hline phi078 & 6 & CAGCACCAGACTACATGACGTGTAA & GGGCCGCGAGTGATGTGAGT \\
\hline bnlg1702 & 6 & TTATCATCAAATGGAGGACACG & AAAGACACACGCTAATGGGC \\
\hline umc2165 & 6 & AGAACACCAAATGGTGACGTTATGT & CTAGCTCGTCTTCCCTGTGGTCT \\
\hline umc1105 & 6 & ATTCCTGCATCATCATCCACTACA & GCCAACTGATCTGCTCTAGCTTC \\
\hline bnlg1154 & 6 & GGGTGATCACATGGGTTAGG & AAATCAATGCTCCAAATCGC \\
\hline nc013 & 6 & AATGGTTTTGAGGATGCAGCGTGG & CCCCGTGATTCCCTTCAACTTTC \\
\hline umc 2332 & 7 & GTCGGAGAAGGAGCTACTGAGCTA & CACAGGTACGTCTGGATGCTGT \\
\hline umc 2325 & 7 & CCTAGGAACTCTGATGGCTATGGA & CTACGATATCCACCTCTACCACCG \\
\hline umc 2364 & 7 & AACCTCAAGATCACCAACATCCTC & CACCCTGCTGTCAGATGGATACTT \\
\hline umc1831 & 7 & TTTCGACTGCTAGTGTACTTGGGG & CTCTACATCTTCAGCGTCTCCACA \\
\hline bnlg1904 & 8 & AGGAGCATGCACTTGGTTCT & ACTCAACTGATGGCCGATCT \\
\hline
\end{tabular}




\begin{tabular}{|l|c|l|l|}
\hline \multicolumn{1}{|c|}{ Marker } & Chrom & \multicolumn{1}{|c|}{ Forward Primer } & Reverse primer \\
\hline phi125 & 8 & ACCGCCGGTGCGAGTTGAAG & CTTGGGATTGCCCTCATCCAC \\
\hline phi087 & 8 & GAGAGGAGGTGTTGTTTGACACAC & ACAACCGGACAAGTCAGCAGATTG \\
\hline umc2134 & 9 & TAGTCTAGCGTCGACGAAAAATGC & CAGGCGACGAAGATGAATTGAA \\
\hline umc1743 & 9 & TGGACTTCGAAAATTCTCTTCAGC & GAGAGGAGGAGCTTCACGAGC \\
\hline umc2133 & 9 & TTCAGGTGTGCACTGACTCTGACT & ATGCTCAAGCTCAACAGCACTTC \\
\hline umc2017 & 9 & AGAGGTTACTACGGAGTGTGGCAG & GTCAGGGTACTGCTTCTCGAACTC \\
\hline umc2021 & 10 & AAACTCAAGCTCGGAATGTACTGC & CGATACTGATCTACTTCACGCTGG \\
\hline umc2126 & 10 & CAGTTCTGCACTTCTGCTTGCTC & AGGACTGTGAAGAGCGCGAG \\
\hline bnlg1 185 & 10 & CGGTCCAGGCAGGTTAATTA & GACTCGAGGACACCGATTTC \\
\hline umc2053 & 10 & ATCTCTCCCTCGCTCTCCTTCTC & AGCAGCAGGTTGGTCGAATG \\
\hline
\end{tabular}


Int.J.Curr.Microbiol.App.Sci (2018) 7(6): 3629-3642

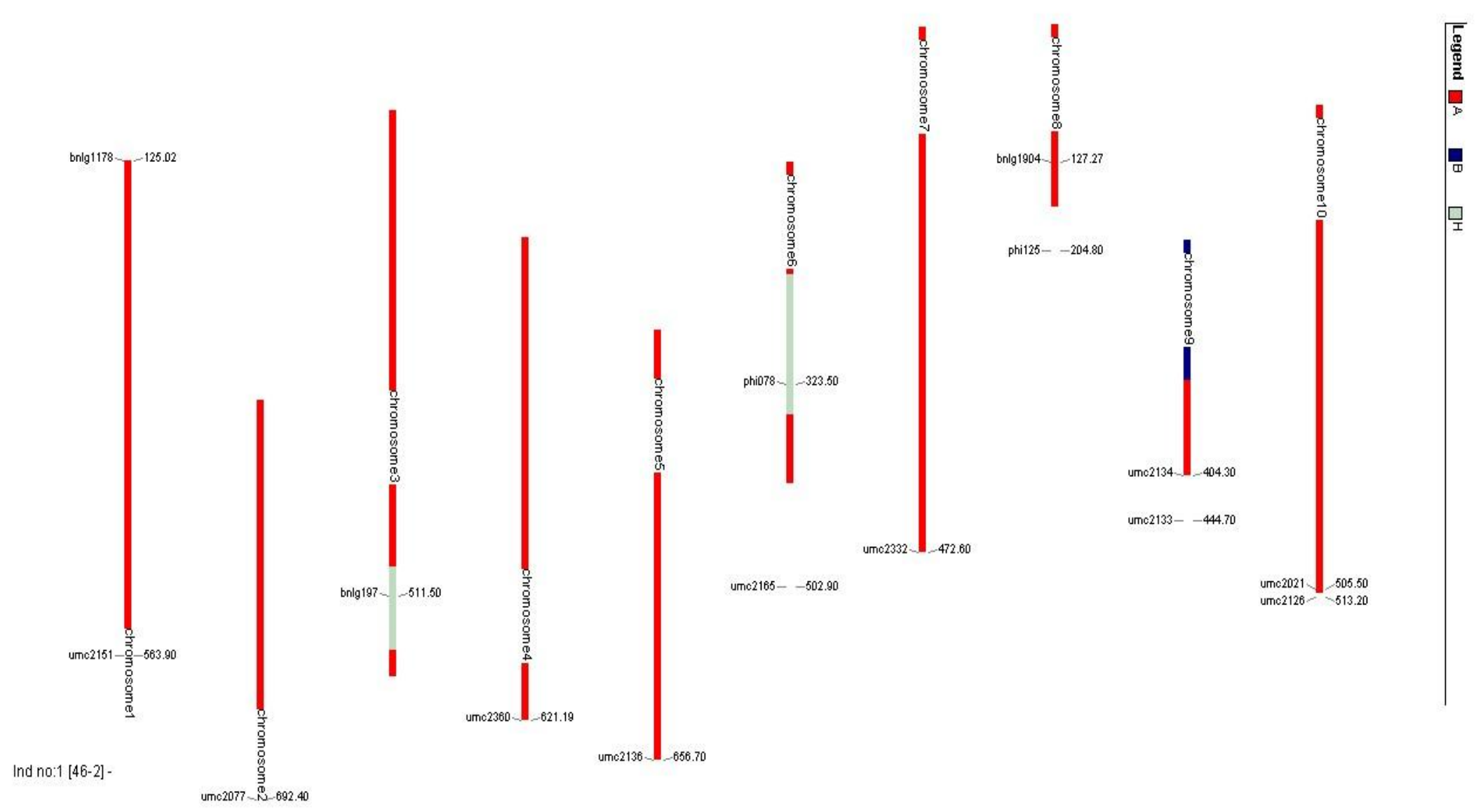

Fig .1 Graphical representation of background screening in developed SDM introgressed line79/936- C1-7-7-7-46-2 
Int.J.Curr.Microbiol.App.Sci (2018) 7(6): 3629-3642

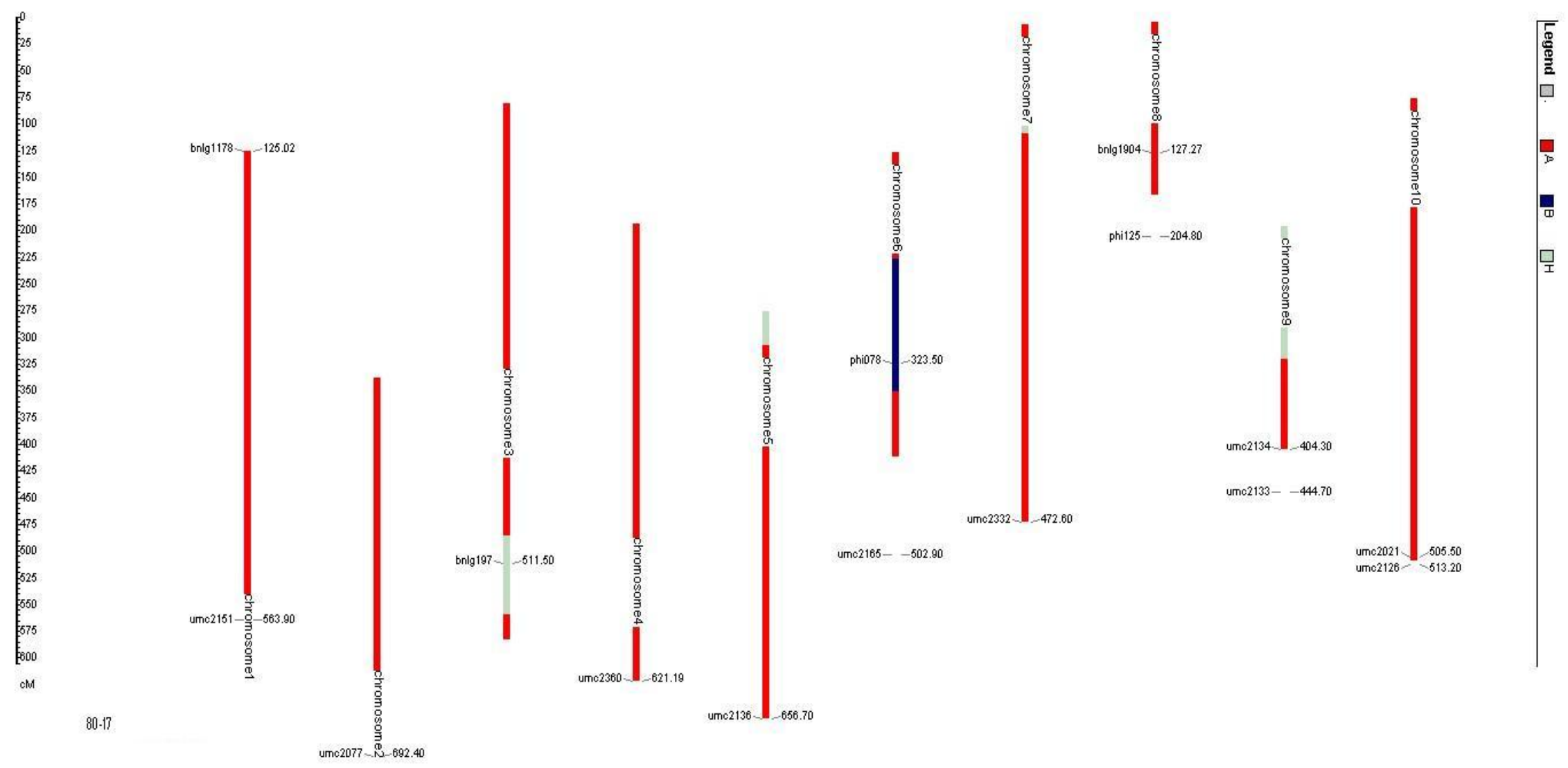

Fig.2 Graphical representation of background screening in developed SDM introgressed line79/936- C1-7-7-7-80-17 
Int.J.Curr.Microbiol.App.Sci (2018) 7(6): 3629-3642
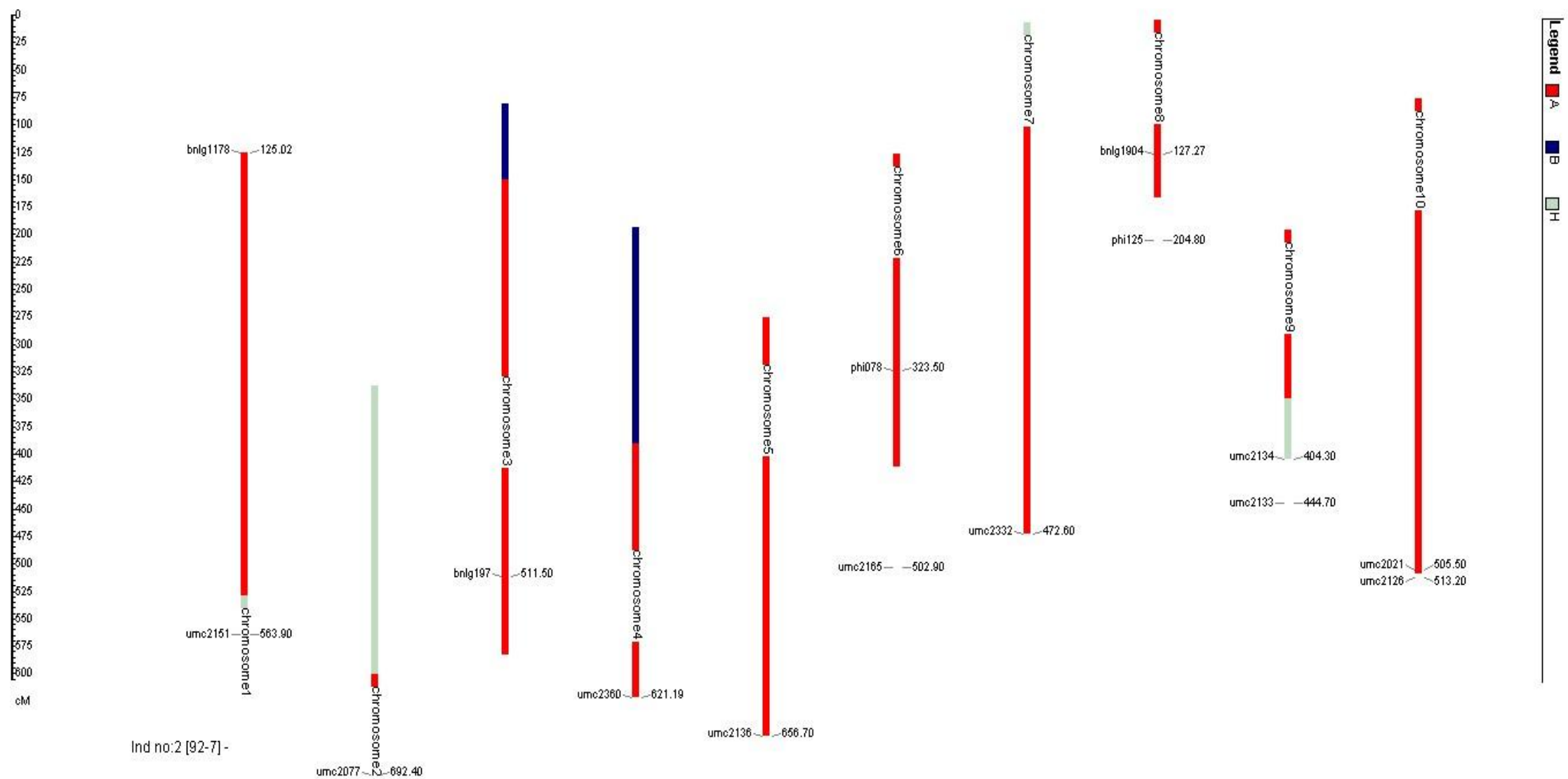

Fig.3 Graphical representation of background screening in developed SDM introgressed line79/936- C1-7-7-7-92-7 
Int.J.Curr.Microbiol.App.Sci (2018) 7(6): 3629-3642

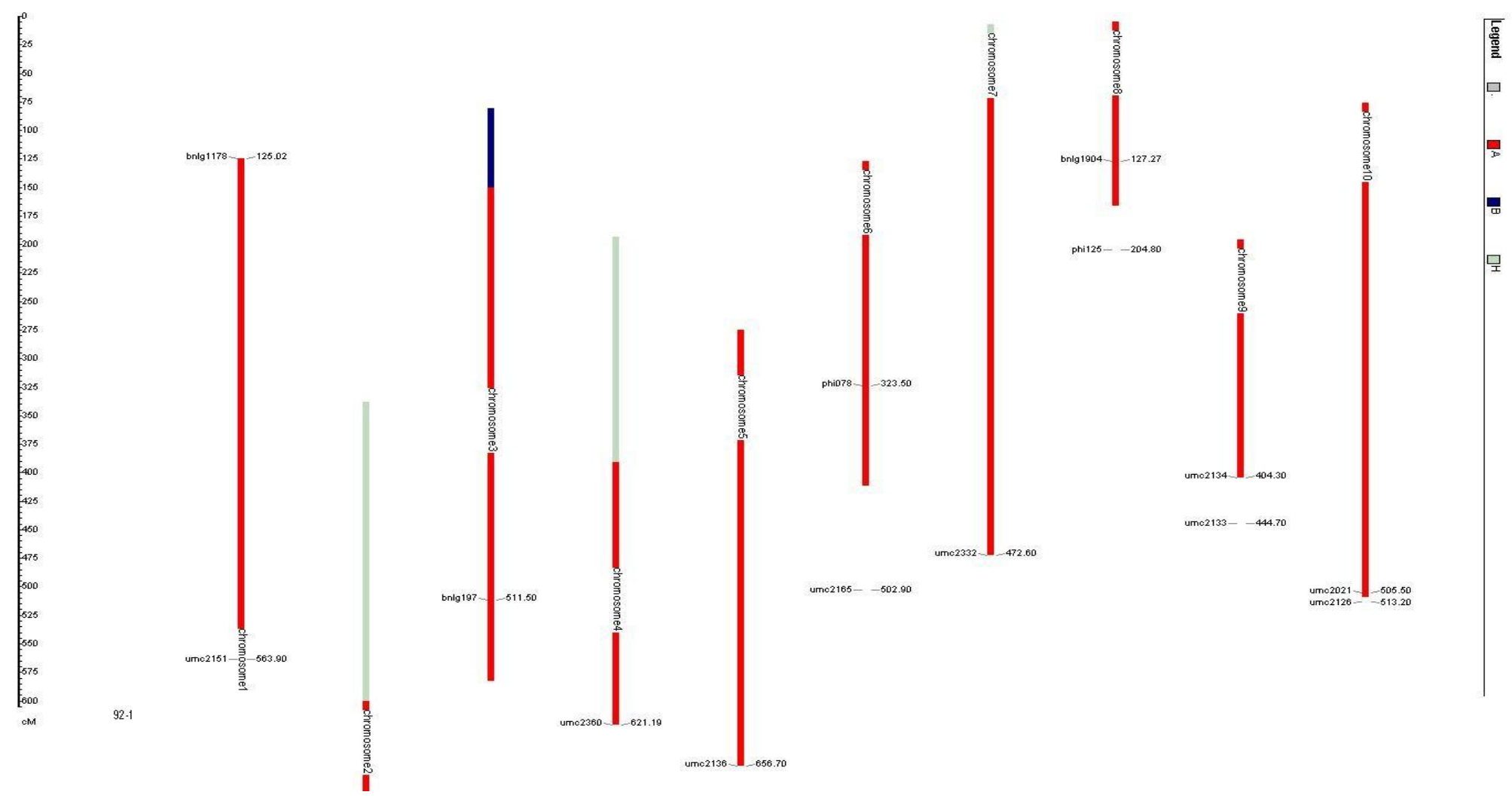

Fig.4 Graphical representation of background screening in developed SDM introgressed line79/936- C1-7-7-7-92-1 


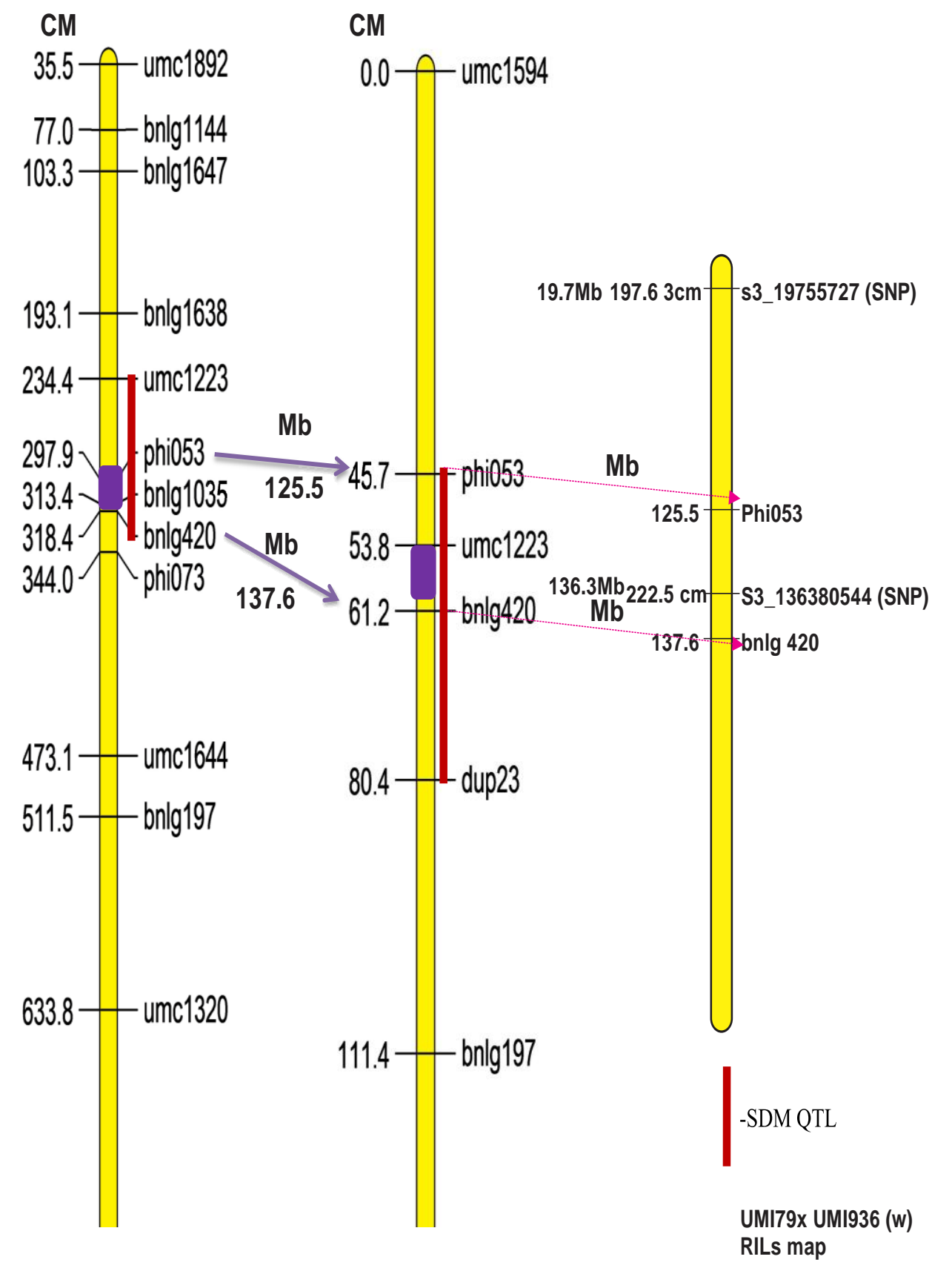

Senthil et al 2015

Personal communication

$\begin{array}{lc}\text { IBM } 2008 \text { Neighbours } 3 & \text { Nair } \text { et al., 2005 } \\ \text { (CM139 x NAI116) }\end{array}$

Markers phi053 and bnlg420 selected for QTLs introgression

Fig.5 Genetic linkage map showing location of SDM QTL on chromosome 3 


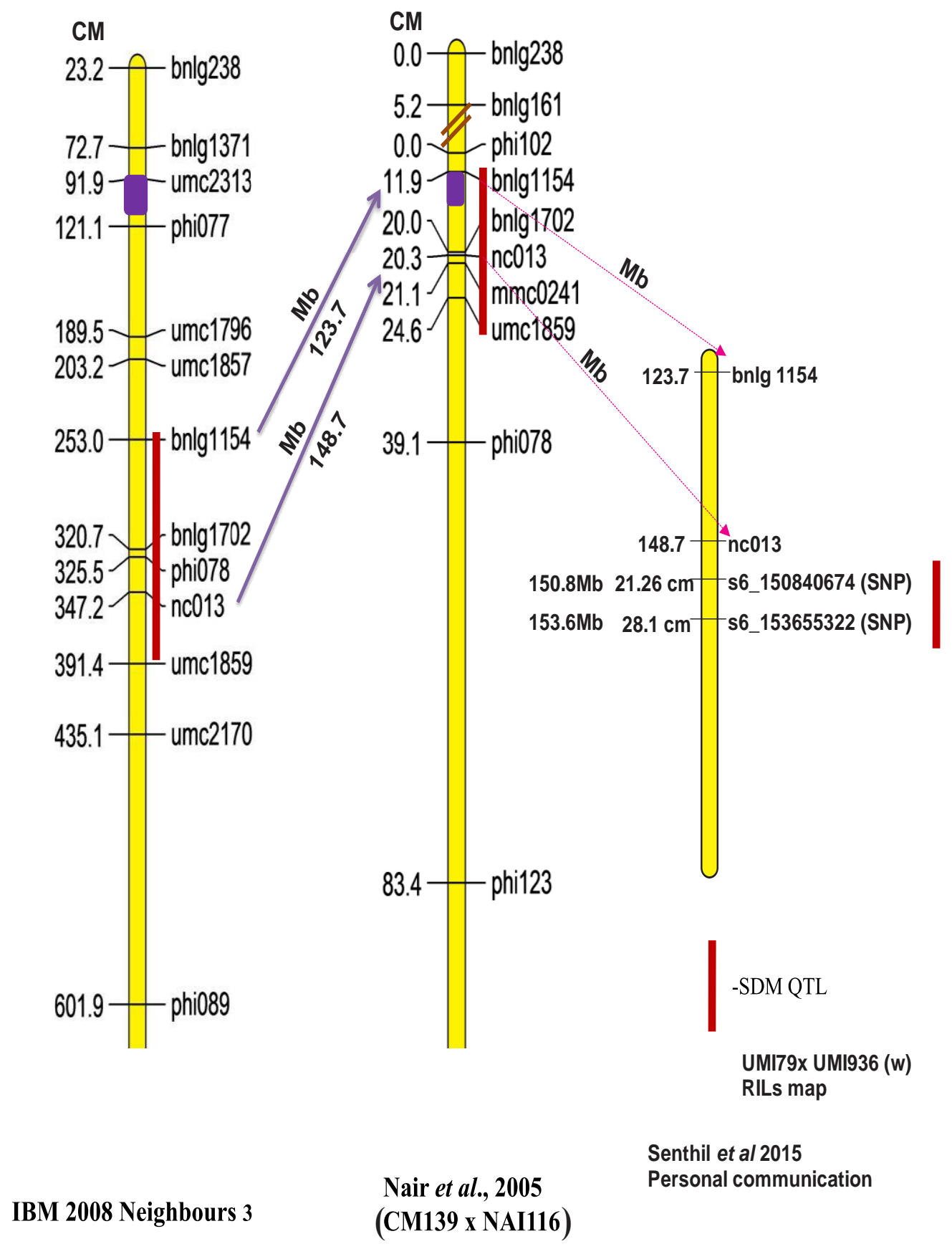

Markers bnlg1154 and nc013 selected for QTLs introgression

Fig.6 Genetic linkage map showing location ofSDM QTL on chromosome 6 


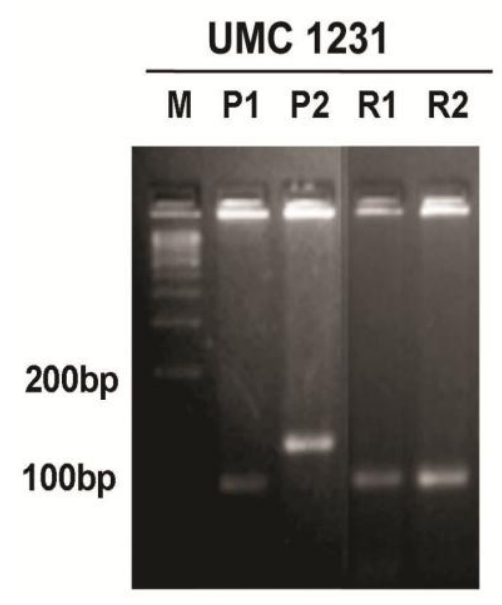

Scoring: $A A$ BB $A A$ AA

$\underline{\text { UMC } 1231}$

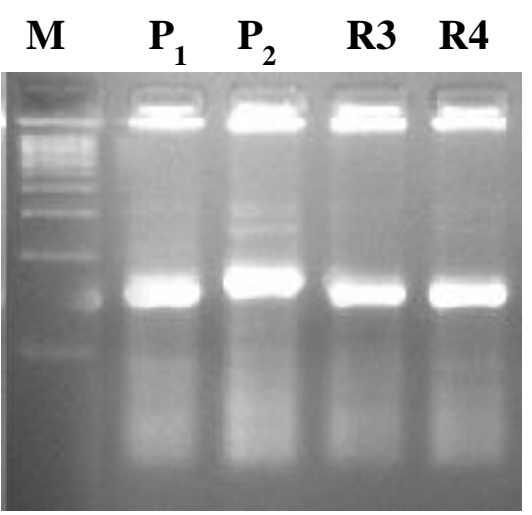

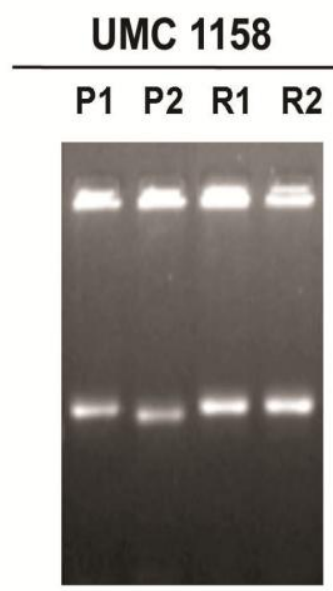

AA BB AA AA

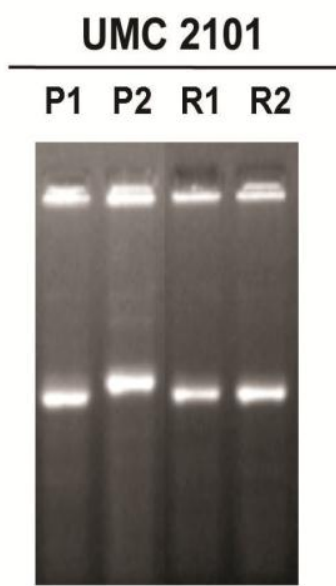

AA BB AA AA

BNLG 1904

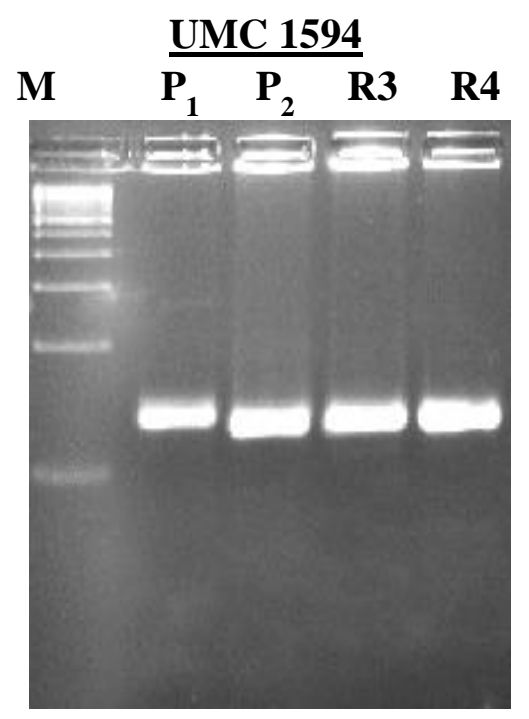

Plate.1 Background screening of SDM introgressed lines $\left(\mathrm{BC}_{3} \mathrm{~F}_{3}\right)$ 
Whereas using markers, it can beachieved by $\mathrm{BC}_{4}, \mathrm{BC}_{3}$ or even $\mathrm{BC}_{2}$ (Visscher et al. 1996, Hospital \&Charcosset1997), thus saving two to four back cross generations.

Background screenings/survey (i.e., profiling the banding pattern of alleles of background polymorphic codominant SSR markers) were done in the SDM resistant plants UMI 79/936-C1-7-7-S7-46-2, UMI 79/936-C1-7-77-80-17, UMI 79/936-C1-7-7-7-92-1 and UMI $79 / 936-C 1-7-7-7-92-7$ in order to assess the recovery of the genome of the recurrent parent. This survey was carried out using 51 polymorphic SSR marker sequences (Supplementary Table.1.) obtained from maizegdb database (www.maizegdb.org). These markers were located among the ten chromosomes of the maize genome. The physical position of the polymorphic markers was obtained from the maizegdb database for each chromosome separately. Parental polymorphism for these two parents UMI79 and UMI 936(w) had already been completed (Plate.19).

\section{Back ground screening of SDM resistant introgressed lines $\left(\mathrm{BC}_{3} \mathrm{~F}_{3}\right.$ generation)}

The 51 SSR markers, which showed distinct alleles between the parents, were selected for the genome wide background analysis. The polymorphic SSR markers were distributed in chromosomes $1,2,3,4,5,6,7,8,9$ and 10 . These markers were used to survey the four SDM resistant $\mathrm{BC}_{3} \mathrm{~F}_{3}$ progenies (i.e., UMI 79/936-C1-7-7-7-46-2, UMI 79/936-C1-7-77-80-17, UMI 79/936-C1-7-7-7-92-1 and UMI 79/936-C1-7-7-7-92-7) for estimating the recovery of the background UMI 79 genotype. The PCR products were screened at 3.5 agarose gel. The plant was scored as AA if it contains UMI 79 allele and scored as BB if it contains UMI 936(w) allele, and scored as $\mathrm{H}$ if it contains both UMI 79 and UMI 936(w) alleles. For example, the analysis of polymorphic markers UMC1231, UMC1158, UMC2101 in parents and two SDM resistant plants are shown in Fig 7.

The graphical genotyping of introgressed lines were performed by using the software GGT 2.0 and the results of graphical genotyping were described.The graphical genotyping of the $\mathrm{BC}_{3} \mathrm{~F}_{3}$ SDMresistant introgressedlines revealed that the complete recovery of UMI 79 genome was present in chromosome 3 in UMI 79/936-C1-7-7-7-46-2 and UMI 79/936-C1-7-7-7-80-17.The complete recovery of UMI 79 genome was present in chromosome 7 in UMI 79/936-C17-7-7-92-1 and UMI 79/936-C1-7-7-7-927.The genotype UMI 79/936-C1-7-7-7-46-2 has revealed that the 92.45 per cent (maximum recovery) of the genome were derived from the recurrent parent UMI 79,genotype UMI 79/936-C1-7-7-7-80-17 andUMI 79/936-C17-7-7-92-7 showed that 89.68 per centand 80.6 per centofrecovery respectively.A minimum 76.8per centofrecovery was observed in the genotype UMI 79/936-C1-77-7-92-1(Fig .24 to Fig 27.). Jorboe et al. (1994) have used the maize genome as a modelfor simulation and reported that three backcross generations and 80 markers were needed to recover 99 per cent of the recurrent parent genotype. Naidoo et al (2012) recovered of the recurrent parent ( 92.15 per cent) in the $\mathrm{BC}_{2} \mathrm{~F}_{1}$ generation in marker-assisted selection for low phytic acid (lpal-1) with single nucleotide polymorphism marker and amplified fragment length polymorphisms for background selection in a maize backcross breeding programme.

To conclude that the percentage of the marker alleles in the four SDM resistant $\mathrm{BC}_{3} \mathrm{~F}_{3}$ progenies that shows similarity with that of recurrent parent is used for calculating the recovery of recurrent parent genome. The genome of UMI 79/936-C1-7-7-7-46-2, UMI 79/936-C1-7-7-7-80-17, UMI 79/936-C1-7-7- 
7-92-1 and UMI 79/936-C1-7-7-7-92-7were 92.45 per cent, 89.68 per cent, 76.8 and 80.06 per cent identical to the recurrent parent respectively.

\section{References}

Hospital, F and A. Charcosset. 1997. Marker assisted introgression of quantitative trait loci. Genet., , 147: 1469-1485.

Jorboe, S.G., W.D. Beavis and Openshaw. 1994. Prediction of re -sponses to selection in marker-assisted backcross programs by computer simulation. Abstracts of the Second Interna-tional Conference on Plant Genome, Scherago International Inc., pp 38.

Naidoo, R., G.M.F. Watson, J. Derera, P. Tongoona and M.D. Laing. 2012. Marker-assisted selection for low phytic acid (lpa1-1) with single nucleotide polymorphism marker and amplified fragment length polymorphisms for background selection in a maize backcross breeding programme. Molecular Breeding. 30:1207-1217.
Paliwal, R. L., G. Granados, H. R. Lafitte, A. D. Violic and J. P. Marathee. 2000. Tropical Maize:Improvement and Production. Food and Agriculture Organization of the United Nations, Rome, Italy.

Prasanna, B. M. and D. Hoisington. 2003. Molecular breeding for maize improvement: an overview. Indian J. Biotech., 2: 85-98.

Visscher, P. M., Chris, S. Haley, M and T. Robin 1996. Marker-Assisted Introgression in Backcross Breeding Programs. Genetics. 144: 1923-1932.

Young, N. D and S.D. Tanksley. 1989. RFLP analysis of the size of chromosomal segments retained around the tm-2 locus of tomato during backcross breeding. Theor. Appl. Genet. 77: 353-359.

Yu,Y.G., G.R. Buss and M.A. Saghai Maroof. 1996. Isolation of a super family of candidate disease resistance genes insoybean based on a conserved nucleotide-binding site. Proc.Natl Acad Sci USA; 93: 11751-6.

\section{How to cite this article:}

Sumathi, K., K. N. Ganesan and Senthil, N. 2018. Studies on Marker Assisted Background Screening of Sorghum Downy Mildew Resistant Introgressed Lines (BC3F3) in Maize. Int.J.Curr.Microbiol.App.Sci. 7(06): 3629-3642. doi: https://doi.org/10.20546/ijcmas.2018.706.427 